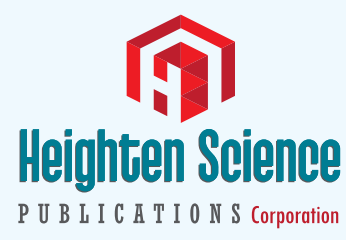

ISSN

2640-2866

\title{
Effects of intraoperative epidural anesthesia during hepatectomy on intraoperative and post-operative patient outcomes
}

\author{
Christopher Mallard', Brad Withers ${ }^{1}$, Brooke Bauer ${ }^{2}$, Paul A \\ Sloan ${ }^{3}$, Sean Dineen ${ }^{4}$ and Annette Rebel ${ }^{5 *}$ \\ ${ }^{1}$ Anesthesia Resident, University of Kentucky, USA \\ ${ }^{2}$ Pain Management Fellow, Baylor Scott and White, USA \\ ${ }^{3}$ Professor, Department of Anesthesiology, University of Kentucky, USA \\ ${ }^{4}$ Assistant Professor, Department of Surgery, University of Kentucky, USA \\ ${ }^{5}$ Professor, Department of Anesthesiology, University of Kentucky, USA
}

\begin{abstract}
*Address for Correspondence: Annette Rebel, Professor of Anesthesiology, University of Kentucky, Medical Center, 800 Rose St. Lexington, KY 40536, USA, Email: arebe2@uky.edu

Submitted: 2 October 2018

Approved: 12 November 2018

Published: 13 November 2018

Copyright: @ 2018 Mallard C, et al. This is an open access article distributed under the Creative Commons Attribution License, which permits unrestricted use, distribution, and reproduction in any medium, provided the original work is properly cited
\end{abstract}

Keywords: Enhanced recovery after surgery; Hepatectomy; Analgesia; Epidural; Neoplasms; Opioid sparing analgesia; Vasopressor

Check for updates

\section{Abstract}

Objective: The objective of this study was to evaluate the effects of intraoperative epidural anesthesia combined with balanced general anesthesia on intraoperative hemodynamics and fluid requirement, and on postoperative patient outcome.

Design: The study design was a retrospective data analysis of patients undergoing open hepatectomy at a single tertiary care center from May, 2013 to June, 2016. Patients undergoing hepatectomies were separated into two groups: patients not receiving epidural local anesthetic intraoperatively (either no epidural or epidural catheter not used intraoperatively) were designated the control group and patients receiving epidural local anesthetic intraoperatively (bolus and/or continuously). Patients were excluded if they underwent laparoscopic or non-elective procedures.

Results: 103 patients were included in the data analysis: Control $n=14$, Epidural $=89$ patients. There were no major differences in demographics between groups. Epidural patients did not have higher requirements in intraoperative intravenous fluid administration, blood loss, or vasopressor use compared to control patients. Patients who received epidurals required less intravenous opioids with better postoperative pain scores initially and also on post-operative day 2 . There were no differences in length of time to ambulation, or post-operative acute kidney injury amongst groups.

Conclusions: This study shows that patients undergoing hepatectomies using combined epidural and general anesthesia: 1) have no increased requirement for intraoperative crystalloid, colloid, or blood component therapy, 2) require lower total intravenous opioid dose, and 3) subjectively report better pain control. Therefore, intraoperative epidural anesthesia combined with general anesthesia may be advantageous for ERAS protocol based oncological procedures.

\section{Introduction}

Enhanced Recovery after Surgery (ERAS) protocols are becoming more frequently used for several surgical procedures after they were first described by colorectal surgeons in the 1990's. The goal of ERAS protocols is to facilitate the early return of patients to normal physiology by minimizing perioperative stressors [1]. In the field of liver surgery randomized clinical trials have proven that ERAS protocols lead to reduced post-operative complications [1].

Epidural anesthesia has been successfully implemented as part of a multimodal approach to pain control in patients undergoing ERAS protocols. Epidural usages 
can provide high quality analgesia while also leading to decreased cardiopulmonary complications, reduced ileus and early mobilization of patients following liver resection [1-3]. However epidural placement and usage is not without complications. This includes sympathectomy induced hypotension, spinal headache, epidural hematomas, epidural abscess or spinal cord injury [3]. Post-operative coagulopathy is expected after liver surgery due a combination of the extent of liver resection, consumptive coagulopathy, and blood loss. The degree of post-operative coagulopathy can be related to preoperative liver synthetic function. Thus, this transient post-operative coagulopathy is typically maximally deranged on postoperative days one and two and typically resolves on its own within five to seven days [3,4]. Therefore, there may be some hesitation by anesthesia providers to use epidural analgesia perioperatively for this procedure. While the coagulopathy may pose an increased risk of epidural hematoma formation patients undergoing hepatectomy and should be monitored prior to epidural catheter removal, it does not prohibit the placement and usage of epidural analgesia $[3,4]$.

Surgical resection is mainstay for a majority of cancer treatment even though excision of the primary tumor can result in further dissemination and growth. Stress from both a surgical and anesthetic point of view has been shown to suppress the immune system [5]. Pain is an obvious inducer of the stress response thus medication to reduce pain and inflammation such as opioids and non-steroidal anti-inflammatories (NSAIDs) can decrease metastatic potential in animal models and humans. Regional anesthesia can dramatically reduce many of these stress response effects of surgery and anesthesia. Studies have shown that patients undergoing abdominal surgery with epidural analgesia had higher numbers of lymphocytes, T-helper cells, preserved interferon- $\gamma$ concentrations and a beneficial Helper T cell ratio [5].

Thus, the practice of using epidural analgesia to decrease pain and inflammation as well as opioid sparing with local anesthesia may play an important role on immune function and cancer recurrence. However, aggressive use of epidural anesthesia in combination with general anesthesia during hepatectomy may have significant impact on hemodynamics and intravenous fluid requirements.

The primary aim of this study was to investigate if the intraoperative use of epidural anesthesia and analgesia combined with general anesthesia is associated with increased use of vasoactive medications or need for more intravenous fluid administration. In addition, the study explored if the epidural use was related to differences in postoperative analgesia or opioid consumption. We hypothesized that epidural anesthesia can be safely used intraoperatively during open hepatectomy and does not interfere with the goals of ERAS protocols for this procedure.

\section{Material and Methods}

After approval from the University of Kentucky Institutional Review Board, a retrospective data analysis was performed on patients undergoing elective open hepatectomy from May, 2013 to June, 2016. The patient collective was separated into two groups: patients not receiving epidural local anesthetic intraoperatively (either no epidural or epidural catheter not used intraoperatively) were designated the control group $[\mathrm{C}]$ and patients receiving epidural local anesthetic intraoperatively [E]. Inclusion criteria into the study was defined as patients undergoing open procedures for removal of either primary liver tumors or with metastases to liver. After excluding patients that underwent laparoscopic procedures, patients with history of liver transplantation or non-elective procedures, we enrolled fourteen control patients and eighty-nine epidural patients. We did not take into account if the patient had cirrhosis or make a distinction between a major or minor hepatectomy in this study.

Due to the small number of control patients compared to those with an epidural, we identified a subgroup of patients in the Epidural group, in which epidural anesthesia was provided as continuous infusion of local anesthetic (lidocaine 1\% or bupivacaine $0.125 \%$ ) intraoperatively as adjunct to general anesthesia ( $\mathrm{gtt}, \mathrm{n}=23$ ). To further 
quantify the association of epidural use and vasopressor requirements, we performed a subgroup analysis (Control [C] $n=14$, Epidural bolus use [E bolus, $n=66$ ], and Epidural continuous infusion [E gtt, $\mathrm{n}=23$ ]. The main difference between $\mathrm{E}$ bolus and $\mathrm{E}$ gtt was that the majority of patients in the $\mathrm{E}$ bolus group did not receive epidural local anesthetics until the final stage of the surgical procedure. The frequency of intraoperative vasoconstrictive medication administration for all groups was recorded.

Data collection was performed in a blinded fashion by a clinical support staff member that was not involved in the analysis of the data before patients were separated in respective groups. Intraoperative data were collected and analyzed for opioid use with all opioids converted to fentanyl equivalents via the Global RPh opioid equivalence calculator [6] and tallied together in Microsoft Excel to determine total dose, frequency of hypotension defined as a mean arterial pressure (MAP) less than $60 \mathrm{mmHg}$ for $>10 \mathrm{~min}$ duration post incision. Vasopressor therapy was defined as either no usage, low dose: less than 500 micrograms (mcg) Phenylephrine, 25 milligrams (mg) ephedrine, or 2 units of vasopressin accumulatively for the procedure, or high dose: any vasopressor infusion, any bolus of epinephrine, any combination of drugs where one drug is a greater than any low parameter.

To investigate the intraoperative implications of continuous epidural anesthesia for intraoperative management during hepatectomy, we reviewed the anesthesia records of patient without epidural use [C] and continuous epidural anesthesia [EPI gtt] and calculated the intraoperative doses of vasoconstrictive medications.

Average total fluid administration including blood transfusion, and estimated blood loss were other intraoperative factors compared between the control and epidural groups. We also recorded the total amount of opioid for the case after converting all drugs and routes to intravenous fentanyl equivalents.

Post-operative data were analyzed for pain scores immediately post op, twentyfour and forty-eight hours postoperatively, length of stay, time to ambulation and rate of acute kidney injury. Data are presented as mean \pm standard deviation. Statistical analysis was performed using a paired T-test and Fisher's Exact test. Statistical significance was assumed if $\mathrm{P}$ value was less than 0.05 .

\section{Results}

Eighty-nine of the one hundred and three patients met our inclusion criteria and there were no significant demographic differences between the two groups: age $(p=$ 0.35), American Society of Anesthesia Class ( $p=0.22)$, male to female ratio $(p=0.09)$ and operating room duration ( $p=0.25$ ) (Table 1$)$. Intraoperative epidural use was not associated with differences in estimated blood loss $(\mathrm{p}=0.33)$, packed red blood cell transfusion ( $p=0.27)$, total fluid administration (crystalloid and colloids) $(p=0.5)$, total intravenous crystalloid fluid ( $\mathrm{p}=0.45)$, and intravenous albumin administration ( $p=0.98$ ). Measured urine output for the case was also compared between the two groups and was found to have no significant difference as well $(p=0.51)$ (Table 2). There were no differences in the requirements and extent of vasopressor therapy between the two cohorts. There was no significant difference in the no vasopressor requirement (E: 33 out of 89 patients, $C: 8$ out of 14 patients; $p=0.27$ ), low vasopressor requirements (E: 23 out of 89 patients, C: 1 out of 14 patients; $p=0.31$ ), and high vasopressor requirement (E: 33 out of 89 patients, $C: 5$ out of 14 patients; $p=0.62$ ) factions between the control and epidural cohorts (Figure 1).

\begin{tabular}{|c|c|c|c|}
\hline \multicolumn{2}{|c|}{ Table 1: Demographics of the study population by method of pain control. Data presented as mean \pm SD. } \\
\hline N & Epidural & Control & p-value \\
\hline Age (years) & 89 & 14 & 0.35 \\
\hline ASA class & $54.2 \pm 12.7$ & $50.7 \pm 10.1$ & 0.22 \\
\hline M:F ratio & $2.9 \pm 0.5$ & $3.0 \pm 0.4$ & 0.09 \\
\hline OR duration (min) & $42: 47$ & $10: 4$ & 0.25 \\
\hline
\end{tabular}


Table 2: Effects of epidural usage on intraoperative fluid requirements and urine output compared to control. Data presented as mean \pm SD.

\begin{tabular}{|c|c|c|c|}
\hline & Epidural & Control & p-value \\
\hline N & 89 & 14 & 0.33 \\
\hline EBL $(\mathrm{mL})$ & $292 \pm 307$ & $455 \pm 585$ & 0.5 \\
\hline Total Fluids $(\mathrm{mL})$ & $2301 \pm 1236$ & $2757 \pm 2436$ & 0.45 \\
\hline IVF Crystalloid $(\mathrm{mL})$ & $1975 \pm 1020$ & $2436 \pm 2166$ & 0.98 \\
\hline IVF Albumin $5 \%(\mathrm{~mL})$ & $325 \pm 400$ & $321 \pm 504$ & 0.27 \\
\hline PRBC transfusion $(\mathrm{units})$ & $0.03 \pm 0.3$ & $0.5 \pm 1.3$ & 0.51 \\
\hline
\end{tabular}

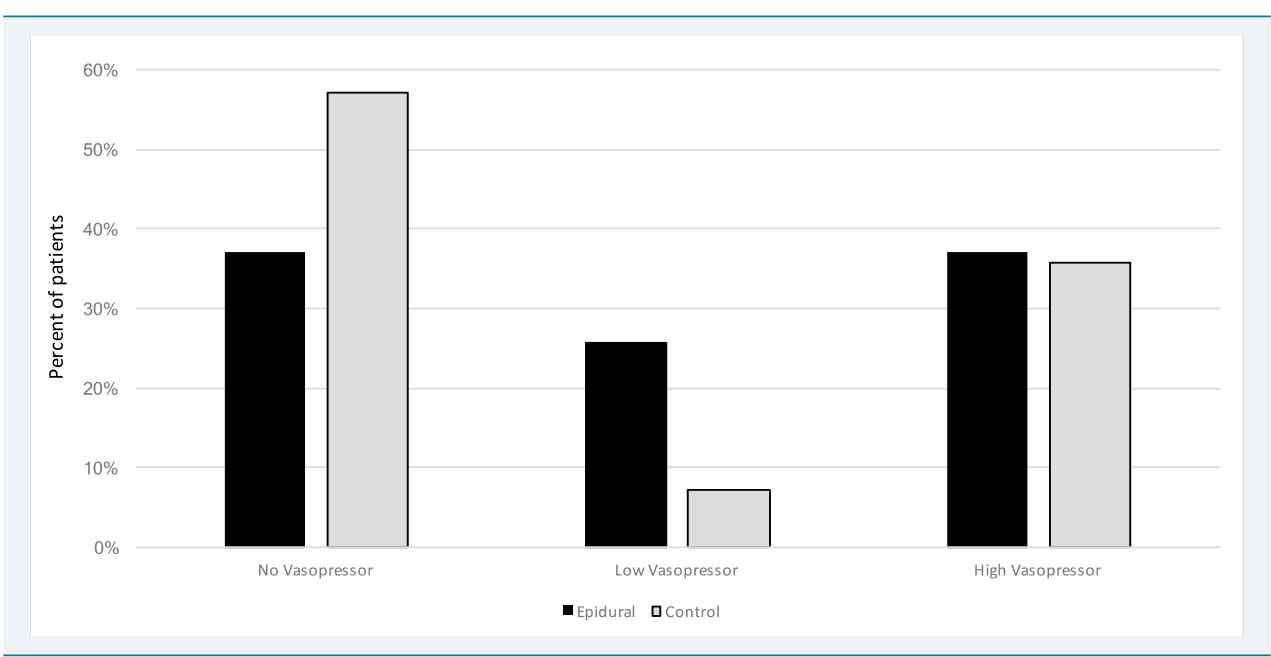

Figure 1: Effects of epidural usage on intraoperative vasopressor frequency and magnitude.

The frequency of vasopressor use was compared in the following groups: patients receiving epidural anesthesia combined with general anesthesia intraoperatively [Epidural, $n=89$ ], were compared to patients without epidural anesthesia or not used intraoperatively [Control, $n=14$ ]

Vasopressors were quantified as no usage, Low $=<500 \mathrm{mcg}$ Phenylephrine, $<25 \mathrm{mg}$ ephedrine, $<2$ units of vasopressin for the case. High = any vasopressor infusion, any bolus of epinephrine or any combination of drugs where one drug is a greater than any low parameter. Data presented as fraction from total.

To obtained more detailed information about vasopressor use and association with intraoperative epidural anesthesia, we identified a subgroup of patients in the Epidural group, in which epidural anesthesia was provided as continuous infusion of local anesthetic (lidocaine $1 \%$ or bupivacaine $0.125 \%$ ) intraoperatively as adjunct to general anesthesia $(\mathrm{E} g \mathrm{tt}, \mathrm{n}=23)$. The frequency of intraoperative vasoconstrictive medication administration is shown in table 3. The frequency of vasoconstrictive medications was not significantly different between Control group and epidural bolus use. There was no difference in low vasopressor requirements between Control patients and patients receiving continuous epidural anesthesia intraoperatively.

For further analysis of intraoperative vasoconstrictive medication administration, we calculated the intraoperative doses of vasoconstrictive medications given to patients in the Control group and to patients with continuous epidural anesthesia (Table 4). The most commonly given agent was phenylephrine. The phenylephrine dose was not different between the groups. The other agents (ephedrine, vasopressin and norepinephrine) were given less frequently and less consistently, most likely representing the preferences of the anesthesia provider. While the data indicated a significantly larger dose of ephedrine in patients with continuous epidural anesthesia, the other, more potent vasoactive medications (Vasopressin and Norepinephrine) were used in both groups in similar concentrations.

In addition to vasopressor use, the subanalysis did not show any differences in intravenous fluid administration between the control group and patients receiving continuous intraoperative epidural anesthesia. In average, patient in the control group received $2757 \pm 2436 \mathrm{ml}$ intravenous fluids (crystalloid $2436 \pm 2169 \mathrm{ml}$, albumin $5 \%$ 
$321 \pm 504 \mathrm{ml})$. The average for patient with intraoperative continuous epidural local anesthesia infusion [E gtt] was $2244 \pm 1330 \mathrm{ml}$ (crystalloid $1859 \pm 1077 \mathrm{ml}$, albumin $5 \% 385 \pm 395 \mathrm{ml}$ ).

When comparing opioid requirements between the control and epidural group, the epidural cohort required significantly less intravenously bolused opioids $286 \pm$ $251 \mathrm{mcg}$ in the epidural group versus $629 \pm 568 \mathrm{mcg}$ in the control group $(\mathrm{p}=0.04)$. However, when comparing the total dose of opioids for the case $426 \pm 251$ mcg in the epidural group versus $629 \pm 568 \mathrm{mcg}$ in the control group this was not significantly different ( $p=0.21$ ) (Figure 2).

Post operatively, pain scores were assessed via the Visual Analog Scale (VAS) initially after surgery, post-operative day one (24hrs postop) and post operatively day two (48hrs postop). There was a significant difference in VAS pain scores at the initial assessment $5.4 \pm 3.5$ in the epidural cohort versus $8.25 \pm 2.0$ in the control $(p<0.01)$. This was shown as well on post-operative day two $4.2 \pm 2.8$ in the epidural cohort and $6.8 \pm 1.5$ in the control cohort $(\mathrm{p}<0.01)$. However, pain scores on postoperative day one were not significantly different between the two cohorts $(p=0.06)$ (Figure 3). The rate of acute kidney injury $(\mathrm{p}=0.64)$, time to ambulation $(\mathrm{p}=0.11)$, time to eating $(\mathrm{p}=$ $0.25)$, time to first bowel movement $(p=0.25)$, and time to discharge $(p=0.46)$ were not different between the two cohorts (Table 5).

Table 3: Frequency of intraoperative vasoconstrictive medication administration.

Control: no epidural anesthesia, E bolus: bolus epidural local anesthetics, E gtt: continuous intraoperative epidural local anesthetic infusion.

Statistical analysis with Fisher exact test ( $p=$ values), ${ }^{*} p<0.05$.

\begin{tabular}{|c|c|c|c|c|c|}
\hline & Control & E bolus & E gtt & & \\
\hline \multirow[t]{2}{*}{$\mathrm{N}$} & 14 & 66 & 23 & & \\
\hline & & & & $\begin{array}{c}P \text { value } \\
C \text { vs } E \text { bolus }\end{array}$ & $\begin{array}{l}P \text { value } \\
C \text { vs E gtt }\end{array}$ \\
\hline No vasopressor use & 8 & 28 & 5 & 0.14 & 0.03 \\
\hline Low vasopressor use & 1 & 16 & 7 & 0.24 & 0.07 \\
\hline High vasopressor use & 5 & 22 & 11 & $\begin{array}{c}0.76 \\
\text { high vs low } \\
0.38\end{array}$ & $\begin{array}{c}0.14 \\
\text { high vs low } \\
0.62\end{array}$ \\
\hline
\end{tabular}

Table 4: IntraoperativeVasopressor doses for selected subgroups.

Control: no epidural anesthesia, E gtt: continuous intraoperative epidural local anesthetic infusion. Data are presented as mean \pm SD. Statistical analysis with paired t-test * $p<0.05$.

\begin{tabular}{|c|c|c|c|c|}
\hline N & & Control & E gtt & P value \\
\hline Phenylephrine & & 14 & 23 & 0.61 \\
\hline Ephedrine & {$[\mathrm{mcg}]$} & $1064.1 \pm 3681.7$ & $635.6 \pm 1230.4$ & 0.04 \\
\hline Vasopressin & {$[\mathrm{mg}]$} & $1.43 \pm 5.35$ & $12.83 \pm 19.47^{\star}$ & 0.75 \\
\hline Norepinephrine & {$[\mathrm{mcg}]$} & $29.57 \pm 110.65$ & $1.05 \pm 2.91$ & 0.30 \\
\hline
\end{tabular}

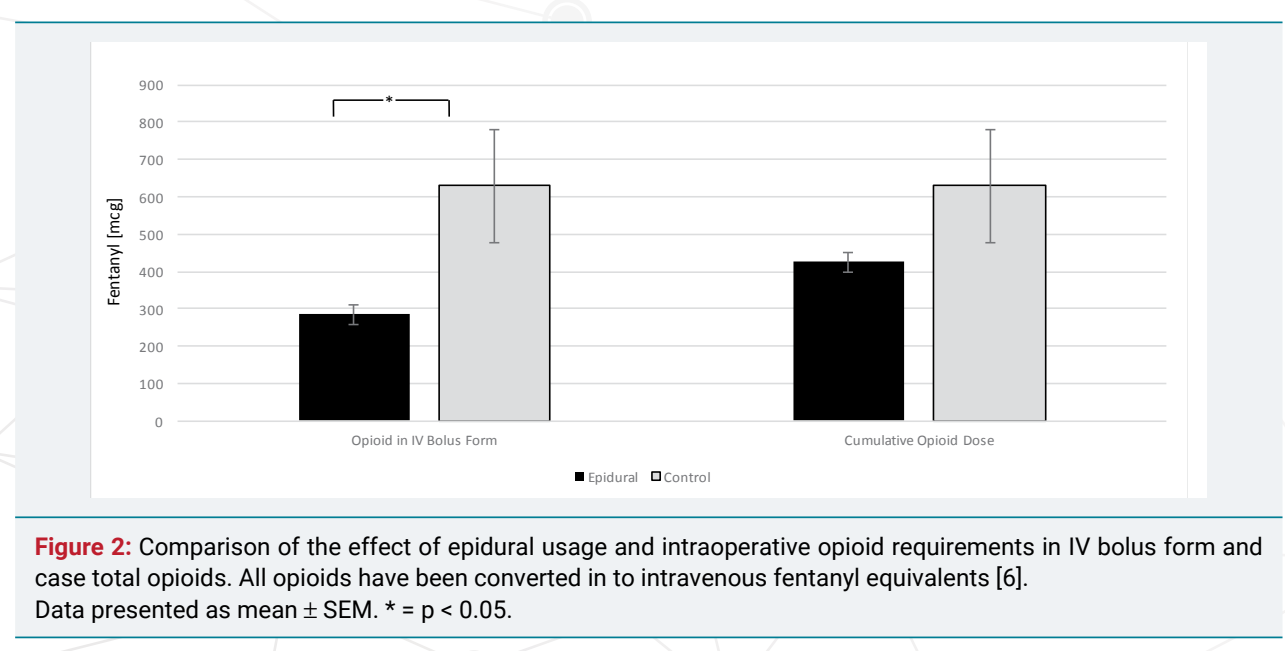




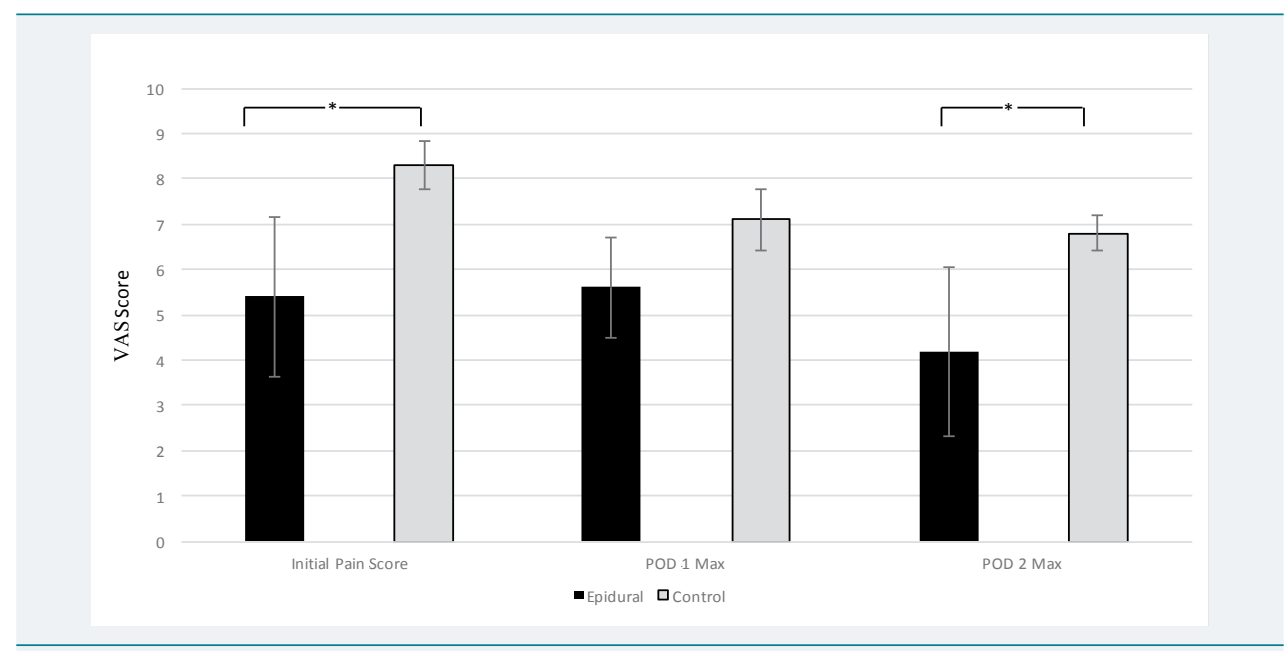

Figure 3: Effects of epidural usage on VAS scores on the initial post-operative assessment, and maximum pain scores on post-operative day 1 and 2 . Data presented as mean \pm SEM. ${ }^{*}=p<0.05$

Table 5: Comparison of post-operative outcomes in patients that received epidural analgesia versus control. Data presented as mean \pm SD.

\begin{tabular}{|c|c|c|c|}
\hline N & Epidural & Control & p-value \\
\hline & 89 & 14 & \\
\hline AKI (percent of patients) & & & 0.64 \\
\hline Time to ambulation (days) & $8.9 \%$ & $7.1 \%$ & 0.11 \\
\hline Time to eating (days) & $2.1 \pm 1.3$ & $2.9 \pm 1.6$ & 0.25 \\
\hline Time to return of bowel function (hours) & $3.6 \pm 1.8$ & $4.9 \pm 4.0$ & 0.21 \\
\hline Time to discharge (days) & $87 \pm 24$ & $97 \pm 35$ & 0.46 \\
\hline
\end{tabular}

The selection of pain control method was not standardized for the patient population and was determined by the surgeon and attending anesthesiologist. Postoperative pain control for rescue was again not standardized as this was at the discretion of the acute pain attending anesthesiologist. Rescue maneuvers that occurred would have included titration of the epidural infusion with continued patient-controlled boluses, addition of intravenous patient-controlled analgesia after removal of the opioid component in the epidural infusion, removal of the epidural catheter if deemed non-functional and utilization of intravenous or oral opioid medications. In the control group post-operative analgesia outside of the post-operative care unit was at the discretion of the attending surgical oncologist. There was no reported epidural hematoma or abscess formation after epidural placement or removal, however there is no data on any delays in catheter removal secondary to coagulopathy.

\section{Discussion}

Epidural usage during abdominal surgeries provides excellent analgesia with reduced cardiopulmonary complications, duration of ileus and opioid requirements however, there is concern over complications associated with their usage and placement $[3,7]$. Liver surgery itself places patients at an increased risk of post-operative coagulopathy and development of epidural hematoma following catheter removal $[3,4]$. Other complications of intraoperative epidural usage in ERAS patients are sympathectomy induced hypotension with concomitant additional fluid requirements and possibly red cell transfusion [1-3].

Our study found that the intraoperative use of epidural anesthesia and analgesia with local anesthetics and opioids in addition to general anesthesia during hepatectomy is not associated with an increased vasopressor use or intravenous fluid administration. The study confirmed that intraoperative epidural use is safe and that the provided analgesia may be superior to intravenous opioid based analgesia, and may offer additional opioid-sparing advantages for patients undergoing oncological resections. 
Epidural induced sympathectomy and concomitant hypotension can lead to increased fluid and vasopressor requirements as a response to the relative hypovolemia. This is a concern in patients undergoing major abdominal surgery with ERAS protocols [1-3]. Our study showed no statistically significant increase in fluid requirements, blood product transfusion or vasopressor usage. Specifically, our study documented that the continuous epidural local anesthesia infusions as an adjunct to balanced general anesthesia did not increase intravenous fluid administration or changed vasopressor requirements, therefore supporting the goals of the ERAS approach. These results are a favorable hemodynamic profile with epidural usage in comparison to the recent literature. Allen et al. [2], showed no significant difference in fluid requirements between their cohort comparing epidural and IVPCA patients undergoing liver resection. However, Siniscalchi et al. [4], found that epidural anesthesia increased the use of intravenous colloid administration. However, they did not find a difference in crystalloid or red cell transfusion. Recorded urine output in this study showed no significant difference between epidural and control patients, this is similar in result to the Siniscalchi et al. [1]. To our knowledge, this is the first study documenting that epidural anesthesia in combination with general anesthesia is not associated with an increased use in vasoconstrictive or inotrope medications during hepatectomy to maintain normotension. While Siniscalchi et al. [1] reported an association of epidural anesthesia with lower MAP post liver resection and at the end of surgery, the authors did not report intraoperative treatment and significance of the hypotension.

This study data indicates that epidural usage intraoperatively leads to decreased bolus IV opioids, however total dose of opioid was not statistically significant. Usage of epidural catheters with local anesthetic and opioid may typically results in opioid sparing effects. This may be beneficial due to studies showing that opioids themselves can result in tumor progression via endothelial cell proliferation and angiogenesis ${ }^{5}$. Non-small cell lung carcinomas for example have been shown to express the muopioid receptor at five to ten-fold higher levels than normal cells. Morphine has been the most studied opioid in this context and has been shown to stimulate endothelial cell proliferation, inhibition endothelial apoptosis resulting in angiogenesis and tumor progression in rat models. However, opioids themselves assist in blocking the stress response which may lead to a favorable immunologic profile in the patient possibly leading to decreased metastasis. This is due in part to pro-inflammatory cytokines decreasing T-cell, B-cell and natural killer (NK) cell function for days following a surgical insult. NK cells play an important role in tumor biology since their activity can be correlated to patient's susceptibility to multiple types of cancers. Studies have shown that decreased NK cell activity from stress induced cytokines results in increased metastasis and when those stress cytokines are blocked we see the reverse effect ${ }^{5}$. This makes a case for further study into long term cancer recurrence in patients undergoing hepatectomies with opioid sparing techniques. However, the data about immunomodulation has been only shown in rat models and no long-term translation studies in humans have been conducted thus far.

In regards to routes of administration when given systemically opioids act on all corresponding receptors throughout the body. Studies have shown that there is synergy between spinal and systemic administration where small doses of spinal morphine significantly potentiated the activity of systemic morphine [8]. In clinical practice, epidural administration of opioids leads to higher concentrations of the drug at the spinal level secondary to their diffusion into the cerebrospinal fluid. Also the epidurally administered opioid is absorbed into the systemic circulation via the highly vascular Batson's venous plexus [9]. This could explain the decreased requirements for bolus IV opioid in our epidural group.

Post-operative outcomes were also assessed and patients that received epidurals had superior pain control immediately post-op as well as at 48 hours based off VAS 
scores. It was also noted that when comparing the two groups there was no difference in the rate of AKI post op as well. However, other post-operative outcomes such as length of stay and time to eating, return of bowel function, ambulation did not show any significant difference. This is an agreement with the Allen et al. [2] study who also was unable to show differences in length of stay or time to eating and Siniscalchi et al. [1], who compared creatinine at post-op days 1, 3 and 7.

Potential limitations of this study are its retrospective nature and the possible addition of selection and/or information biases. We were reliant upon the documentation in the medical record as accurate and precise with proper standardization. However, there is the opportunity for the variable to either be missing, delayed or incomplete. There was also no standardized anesthetic plan for patients undergoing elective hepatectomies and thus some patients received other medications both preoperative and intraoperatively which may have an effect on the results of this study. The patient cohorts were at a single university hospital and thus unable to compare patients undergoing the same procedure in other areas of the country. We also are unable to evaluate the long-term impact on patient morbidity and mortality to evaluate for long term outcomes of opioid sparing techniques.

This study shows that patients undergoing hepatectomies using combined epidural and general anesthesia had no increased intraoperative requirements for intravenous fluid and vasopressor usage and required a lower total intravenous opioid dose perioperatively for adequate pain control. When comparing postoperative outcomes, patients with epidurals had better pain control without a difference in post-operative complications. Although we did not find differences in length of stay, time to ambulation, or time to tolerating oral intake, future studies are needed to explore any advantages of opioid sparing anesthesia techniques for patients undergoing hepatectomy. Thus, with the advent of ERAS protocols and fast track liver surgery combined epidural and general anesthesia is safe and possibly more beneficial outcomes given the decreased IV opioid requirements.

\section{Acknowledgment}

This research was presented at the Kentucky Society of Anesthesiologists annual meeting on 3/18/2017 in Lexington, Kentucky and the Internal Anesthesia Research Society Meeting on 5/7/17 in Washington, D.C.

\section{References}

1. Siniscalchi A, Gamberini L, Bardi T, Laici C, Gamberini E, et al. Role of epidural anesthesia in a fast track liver resection protocol for cirrhotic patients - results after three years of practice. World $J$ Hepatol. 2016; 8: 1097-1104. Ref.: https://goo.gl/HWzZSG

2. Allen S, DeRoche A, Adams L, Slocum KV, Clark CJ, et al. Effect of epidural compared to patientcontrolled intravenous analgesia on outcomes for patients undergoing liver resection for neoplastic disease. J Surg Oncol. 2017; 115: 402-406. Ref.: https://goo.gl/JE14wk

3. Tzimas P, Prout J, Papadopoulos G, Mallett SV. Epidural anaesthesia and analgesia for liver resection. Anaesthesia. 2013; 68: 628-635. Ref.: https://goo.gl/3mB1y7

4. Elterman KG, Xiong Z. Coagulation profile changes and safety of epidural analgesia after hepatectomy: a retrospective study. J Anesth. 2015; 29: 367-372. Ref.: https://goo.gl/5r9McC

5. Tedore $\mathrm{T}$. Regional anaesthesia and analgesia: relationship to cancer recurrence and survival. $\mathrm{Br} \mathrm{J}$ Anaesth. 2015; 115 Suppl 2: ii34-45. Ref.: https://goo.gl/Hq3CTH

6. McAuley D. Global Rph Opioid analgesic converter. Ref.: https://goo.gl/mRWPxr

7. Schreiber KL, Chelly JE, Lang RS, Abuelkasem E, Geller DA, et al. Epidural Versus Paravertebral Nerve Block for Postoperative Analgesia in Patients Undergoing Open Liver Resection: A Randomized Clinical Trial. Reg Anesth Pain Med. 2016; 41: 460-468. Ref.: https://goo.gl/smiHux

8. Pasternak GW. Opiate pharmacology and relief of pain. J Clin Oncol. 2014; 32: 1655-1661. Ref.: https://goo.gl/8K9hET

9. Pasternak GW, Pan YX. Mu opioids and their receptors: evolution of a concept. Pharmacol Rev. 2013; 65: 1257-1317. Ref.: https://goo.gl/BRPPLL 